\title{
Expression stability of six housekeeping genes: a proposal for resistance gene quantification studies of Pseudomonas aeruginosa by real-time quantitative RT-PCR
}

\author{
Hakan Savli, ${ }^{1}$ Aynur Karadenizli, ${ }^{2}$ Fetiye Kolayli, ${ }^{2}$ Sibel Gundes, ${ }^{3}$ \\ Ugur Ozbek ${ }^{4}$ and Haluk Vahaboglu ${ }^{3}$ \\ ${ }^{1-3}$ Tibbi Biyoloji $A D^{1}$, Mikrobiyoloji ve Klinik Mikrobiyoloji $A D^{2}$ and Enfeksiyon Hastaliklari ve Klinik \\ Mikrobiyoloji $A D^{3}$, Kocaeli Universitesi, Tip Fakultesi, Sopali Ciftligi, 41900 Kocaeli, Turkey \\ ${ }^{4}$ Genetik AD, DETAE, Istanbul Universitesi, Istanbul, Turkey
}

Correspondence

Haluk Vahaboglu

vahabo@hotmail.com

Received 22 November 2002

Accepted 28 January 2003
Constantly expressed genes are used as internal controls in relative quantification studies. Suitable internal controls for such studies have not yet been defined for Pseudomonas aeruginosa. In this study, the genes $a m p C, f a b D$, pro $C, p b p-2, r p o D$ and $r p o S$ of $P$. aeruginosa were compared in terms of expression stability by real-time quantitative RT-PCR. A total of 23 strains with diverse resistance phenotypes were studied. Stability of expression among the housekeeping genes was assessed on the basis of correlation coefficients, with the best-correlated pair accepted as being the most stable one. Eventually, proC and $r p o D$ formed the most stable pair $(r=0.958 ; P<0.001)$. Next, in four ciprofloxacin-selected $n f x C$-like mutants, levels of oprD, oprM and oprN mRNA were compared with those of their wild-type counterparts. The comparison was made after correcting the raw values by the geometric mean of the internal control genes $p r o C$ and $r p o D$. The level of oprN mRNA was significantly up-regulated, while the oprD gene was down-regulated (although this difference was statistically insignificant), in the mutants. This expression pattern was consistent with that of the expected expression profile of $n f x C$-type mutants; this experiment therefore lends further support to the use of proC and $r p o D$ genes simultaneously as internal controls for such studies.

\section{INTRODUCTION}

Pseudomonas aeruginosa is an important nosocomial pathogen, particularly in intensive care units (Yucesoy et al., 2000). Members of this species are inherently resistant to a range of antibiotics. In addition, they are capable of conferring resistance to others by shifting the regulation levels of various innate genes (Livermore, 1992).

Porins (particularly porin D) and efflux pumps of the resistance-nodulation-division (RND) family are the currently recognized genetic systems that are associated significantly with antibiotic resistance (Okamoto et al., 2001). The exact links between the expression patterns of these and other resistance phenotypes have not been fully elucidated, and inconsistent conclusions appear in the literature (Masuda et al., 2000; Morita et al., 2001; Sumita \& Fukasawa, 1996; Trias et al., 1989). Therefore, studies focusing on this topic still attract considerable interest.
Levels of expression of these genes have traditionally been studied by Western blotting with the aid of mAbs. Unfortunately, this method is unable to help in studying different proteins simultaneously and so is weak in relative comparisons. Moreover, mAbs are not commercially available. The quantification of mRNAs by real-time RT-PCR has been used with great success in other fields (Johnson et al., 2000; Wang \& Brown, 1999). We suggest that this highly sensitive method may also be useful in relative comparison of resistance gene expression.

The reliability of a relative comparison depends largely on the normalization of unwanted variations between samples. Constantly expressed genes, often selected from among housekeeping genes, are used as internal controls for normalization of the results. The proportion of mRNA of constantly expressed genes in the total cellular RNA is assumed to be equal between different samples. Normalization of raw values by means of internal controls therefore serves to eliminate sample-to-sample variation of the RNA isolation and reverse transcription steps and, even more importantly, serves to eliminate variations in total transcriptional activity between cells. 
For this purpose, six housekeeping genes were compared in this study. These were pyrroline-5-carboxylate reductase (proC), malonyl CoA : acyl carrier protein (ACP) transacylase $(f a b D)$, sigma factors RpoD $(r p o D)$ and RpoS (rpoS), penicillin-binding protein $2(p b p-2)$ and chromosomal betalactamase $(\operatorname{ampC})$. The expression stability of these housekeeping genes was first investigated as proposed by Vandesompele et al. (2002) and, later, the levels of oprM, $o p r N$ and $\operatorname{oprD}$ mRNA were compared in a set of $P$. aeruginosa strains.

\section{METHODS}

Strains, resistance tests and beta-lactamase assays. We selected 17 P. aeruginosa strains from a set obtained from university hospitals in four different geographical regions of Turkey. The bacteria had been identified in those universities by various identification systems. The strains were, nevertheless, reidentified in our institute by classical methods and, if required, by the non-fermenter ID panel of the Becton Dickinson system (Diagnostic Instrument Systems).

Of the 17 strains selected, 10 were fully susceptible to antibiotics of the main classes, while the others were of different resistance phenotypes. We challenged the susceptible strains in Mueller-Hinton (MH) broth with ciprofloxacin $\left(0 \cdot 1 \mu \mathrm{g} \mathrm{ml}^{-1}\right)$ in order to obtain resistant variants. Consequently, six isogenic mutants with various resistance patterns were selected. These mutants, as well as their counterparts, were included in the study group.

Resistance patterns were determined by the disk diffusion method on $\mathrm{MH}$ agar plates. MICs were determined by either E-test strips on $\mathrm{MH}$ agar plates or by the agar dilution method as described by the NCCLS. Antibiotic disks and $\mathrm{MH}$ agar were obtained from Oxoid while E-test strips were sourced from AB Biodisc. Powder forms of the antibiotics were obtained as gifts from the respective companies.

Beta-lactamases were analysed as described elsewhere (Vahaboglu et al., 1998). Extracts obtained by freezing and thawing of dense bacterial suspensions were applied to polyacrylamide gels with ampholytes ranging from $\mathrm{pH} 3$ to 10 . Nitrocefin overlay and migration relative to TEM-1 and SHV-1 standards enabled us to evaluate the pI values of beta-lactamases. Precise identification of the beta-lactamases depended on sequence analysis of PCR products as described previously (Vahaboglu et al., 1998).

Random amplified polymorphic DNA (RAPD) typing. Clonal variability was further ensured by RAPD typing of eight selected strains, two from each region. DNA was isolated from fresh overnight agar cultures. A loopful of bacteria was homogenized in $50 \mu \mathrm{l}$ TE buffer ( 10 $\mathrm{mM}$ Tris/HCl, $1 \mathrm{mM}$ EDTA, $\mathrm{pH}$ 8) and lysis was accomplished with $500 \mu \mathrm{l}$ guanidium thiocyanate $(6 \mathrm{M})$ plus $0 \cdot 5 \%$ sodium lauroylsarcosine for $10 \mathrm{~min}$ at room temperature. DNA was extracted first by phenol/chloroform and then by chloroform/isoamyl alcohol (24:1, $\mathrm{v} / \mathrm{v})$ and then precipitated with $0 \cdot 1$ vols sodium acetate $(3 \mathrm{M}, \mathrm{pH} 5 \cdot 4)$ plus an equal volume of 2-propanol at room temperature. Precipitates were collected by centrifugation ( $10 \mathrm{~min}$ at $12000 \mathrm{~g}$ ) and then the pellets were washed twice with $70 \%$ ethanol, air-dried for $2 \mathrm{~min}$ and resuspended in $30 \mu \mathrm{l}$ double-distilled water.

RAPD PCR was performed as described elsewhere (Kerr et al., 1995; Renders et al., 1996). The primers were ERIC-1R (5'-AAGCTCCTGG GGATTCA-3') and ERIC-2 (5'-AAGTAAGTGACTGGGGTGAGCG$\left.3^{\prime}\right)$. Master mixtures were prepared as described in the above references. However, the amplification program was modified as follows: one cycle of denaturation for $5 \mathrm{~min}$ at $95{ }^{\circ} \mathrm{C}$ followed by 25 cycles of $3 \mathrm{~min}$ at $39^{\circ} \mathrm{C}$ (with an increase of $0.3{ }^{\circ} \mathrm{C}$ every cycle), 2 min at $72{ }^{\circ} \mathrm{C}$ and $1 \mathrm{~min}$ at $94{ }^{\circ} \mathrm{C}$ and 30 cycles of $2 \mathrm{~min}$ at $44^{\circ} \mathrm{C}, 3 \mathrm{~min}$ at $72{ }^{\circ} \mathrm{C}$ and $1 \mathrm{~min}$ at $94{ }^{\circ} \mathrm{C}$. A final extension for $1 \mathrm{~h}$ at $72{ }^{\circ} \mathrm{C}$ completed the procedure.

PCR products were separated on a $2 \%$ agarose gel and visualized by ethidium bromide staining. For better resolution, they were also run on a $6 \%$ acrylamide/bis-acrylamide gel (data not shown). The banding patterns of both gels were analysed by the freely distributed gel analysis software LabImage (version 2.62). Molecular sizes of the bands were calculated by this software relative to the marker DNA.

RNA isolation and reverse transcription. Total RNA was isolated from $5 \mathrm{ml}$ fresh overnight (approx. $18 \mathrm{~h}$ ) broth culture ( $\mathrm{MH}$ broth) by using the NucleoSpin RNA II kit (Macherey-Nagel), as described by the manufacturer. Genomic DNA was eliminated by RNase-free DNase I treatment during the isolation procedure. Finally, RNAs were eluted from the silica membranes in a volume of $40 \mu \mathrm{l}$ diethyl pyrocarbonatetreated double-distilled water. The $A_{260}$ of the resulting RNA solution was between 1 and 10 . Reverse transcription was performed at $42{ }^{\circ} \mathrm{C}$ for 90 min by using random hexamer primers so as to obtain cDNA copies of mRNAs $(2 \mu \mathrm{l})$ with $100 \mathrm{IU}$ MMuLV reverse transcriptase (MBI Fermentas) in $20 \mu \mathrm{l}$ total volume. Concentrations of cDNAs were adjusted on a LightCycler (Roche Diagnostics). For every sample, $1 \mu \mathrm{l}$ cDNA and $9 \mu \mathrm{l}$ SYBR Green I (the same concentration as indicated by the manufacturer for the PCR assay) were mixed in capillary tubes. After incubation at $95{ }^{\circ} \mathrm{C}$ for $5 \mathrm{~min}$, fluorescence emissions were read at $55^{\circ} \mathrm{C}$ with the real-time fluorometry facility of the LightCycler. This enabled us to compare the total cDNA concentrations of the samples with the control transcript, which was approximately $1 \mathrm{mg} \mathrm{ml}^{-1}$ at the highest dilution. Concentrations of cDNAs of the samples were adjusted to a level close to the second dilution $\left(10^{-1}\right)$ of the control cDNA. This adjustment was critical for performing successful calculations. The aim was to keep the cDNA concentrations of the samples between the concentrations of the controls in order to avoid large variations during calculations by the LightCycler software.

Real-time PCR. The sequences of the genes studied were obtained from GenBank and the primers were designed with the aid of the OLIGO software (version 5.0; Molecular Biology Insights). The sequences of the primers are shown in Table 1.

PCR was performed in the LightCycler in capillary glass tubes with the LightCycler FastStart DNA Master SYBR Green I kit (Roche). Work was always carried out on desktop coolers (pre-cooled to $4{ }^{\circ} \mathrm{C}$ ). Master mixtures were prepared exactly as recommended by the manufacturer, except for the concentration of $\mathrm{Mg}^{2+}$. The final concentrations of $\mathrm{Mg}^{2+}$ and primers were respectively $2.5 \mathrm{mM}$ and $50 \mathrm{pmol}$ per reaction.

The control cDNA was from $P$. aeruginosa ATCC 27853 and the primers of the control reactions were specific for $p b p-2$. An arbitrary concentration value of $1.5 \times 10^{4}$ copies of the $p b p-2$ gene was assigned to the control transcript. Tenfold dilutions of this down to 15 copies of the $p b p-2$ gene were always included in the reactions.

PCR was accomplished after a 5 min activation and denaturation step at $95^{\circ} \mathrm{C}$, followed by 45 cycles of $15 \mathrm{~s}$ at $95^{\circ} \mathrm{C}, 10 \mathrm{~s}$ at $60^{\circ} \mathrm{C}$ and $15 \mathrm{~s}$ at $72{ }^{\circ} \mathrm{C}$.

Primer dimers and other artefacts were evaluated by melting curve analysis and eventually only dimer- and artefact-free reactions were considered valid. Results were read with the 'second derivative maximum' algorithm of the software provided. The LightCycler software generated a standard curve by plotting 'crossing cycle number' versus logarithms of the given concentrations for each control. Eventually, a regression line was drawn between these points. The software calculated the concentrations of the studied genes with the aid of this standard curve.

Statistical analysis. The stability of mRNA expression was assessed by 
Table 1. Primers used in quantification studies

\begin{tabular}{|lll|}
\hline Target & \multicolumn{1}{c|}{ Forward $\left(\mathbf{5}^{\prime} \boldsymbol{\rightarrow} \mathbf{3}^{\prime}\right)$} & \multicolumn{1}{c|}{ Reverse $\left(\mathbf{5}^{\prime} \rightarrow \mathbf{3}^{\prime}\right)$} \\
\hline$a m p C$ & AGATTCCCCTGCCTGTGC & GGCGGTGAAGGTCTTGCT \\
$f a b D$ & GCATCCCTCGCATTCGTCT & GGCGCTCTTCAGGACCATT \\
$o p r D$ & TCCGCAGGTAGCACTCAGTTC & AAGCCGGATTCATAGGTGGTG \\
$o p r M$ & CCATGAGCCGCCAACTGTC & CCTGGAACGCCGTCTGGAT \\
$o p r N$ & GCGCGAGAAGATTGCCCTGAG & GCGGCGAAAGGTCCACTGTCA \\
$p b p-2$ & CCGCCACTACCCGCTGAAG & TGCCGTGCAACTCGCTCTC \\
$p r o C$ & CAGGCCGGGCAGTTGCTGTC & GGTCAGGCGCGAGGCTGTCT \\
$r p o D$ & GGGCGAAGAAGGAAATGGTC & CAGGTGGCGTAGGTGGAGAA \\
$r p o S$ & CTCCCCGGGCAACTCCAAAAG & CGATCATCCGCTTCCGACCAG \\
\hline
\end{tabular}

calculation of Spearman's correlation coefficients of the raw concentration data with the aid of the statistical package SPSS (version 9.0). The best-correlated pair was considered to be the most stable one.

Stability was further evaluated by a freely distributed MS Excel application (geNorm). Detailed information on this application can be obtained from Vandesompele et al. (2002). This approach assumes that minimally regulated, stably expressed genes stay in a constant ratio to each other. In other words, in a given set of genes, it must be the pair of most stable genes that will be able to keep the ratio to each other constant in different samples. Importantly, co-regulated genes are exceptions to this assumption and they are not included.

The applet geNorm helps to calculate the gene expression stability measure $(\mathrm{M})$, which is the mean pair-wise variation for a gene from all other tested control genes (Vandesompele et al., 2002). A higher value of $\mathrm{M}$ means greater variation in expression. The stepwise exclusion of genes with the highest $M$ values allows the ranking of the tested genes according to their expression stability. The proposed threshold for eliminating a gene as unstable was an expression stability measure of $\geqslant 0 \cdot 5$.

Raw quantities were corrected by dividing a value by the geometric mean of proC and rpoD genes of the same sample. Relative comparisons were done between corrected values with the ANOVA test for significance.

\section{RESULTS AND DISCUSSION}

A total of 23 strains were included in the study. The group was composed of 17 wild-type strains and six ciprofloxacinselected, isogenic mutants. Clonal heterogeneity was investigated by DNA typing of eight of these wild-type strains. One sample did not provide a readable pattern, probably because of inhibitors carried over from the DNA isolation step. However, the other seven were amplified successfully and the fingerprints were sufficiently polymorphic to confirm the clonal diversity (Fig. 1).

Of the 17 wild-type strains, seven were already resistant to various antibiotics. It is noteworthy that three of these did not produce a beta-lactamase other than the chromosomal one (Table 2). These strains were probably resistant because of activated porin and/or efflux systems. Of the six ciprofloxacin-selected mutants, two were resistant only to the fluoroquinolones, while the other four expressed $n f x C$-like multiple-resistance phenotypes (Table 3 ). In addition to fluoroquinolones, the MIC of imipenem increases among

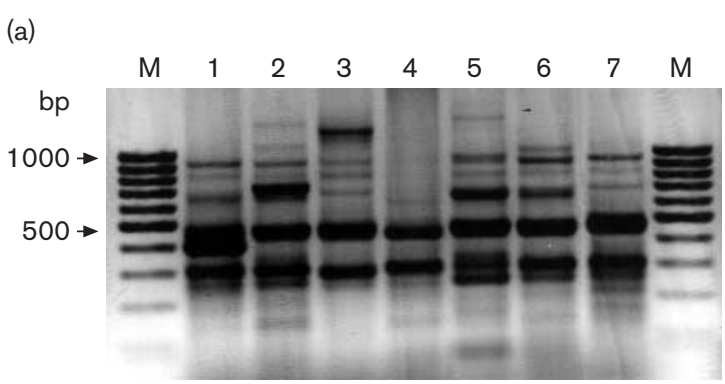

(b)

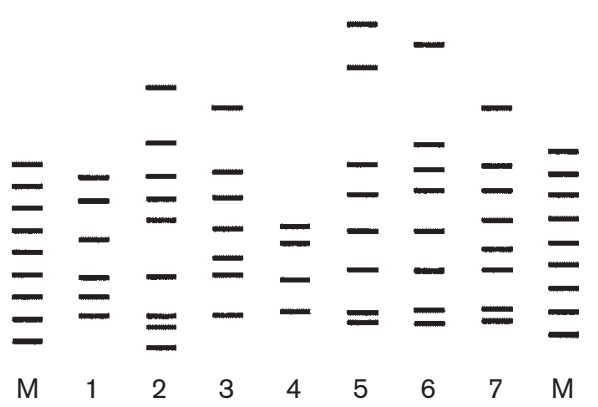

Fig. 1. (a) RAPD fingerprints (negative exposure). Lanes: $M$, molecular size marker (100 bp DNA ladder); $1-7$, selected strains (strains 2 and $5-7$ are the parents of $n f x C$ mutants). (b) Fingerprints according to the lengths (bp) of fragments calculated by gel analysis software. Lanes are the same as in (a).

$n f x C$ mutants because of the concurrent down-regulation of oprD (Maseda et al., 2000; Ochs et al., 1999). The resistance phenotypes of the mutants were in agreement with this.

Interestingly, the MICs of ceftazidime were variable; one was increased and one decreased while the other two were unchanged. One study reported an unexplained decrease in the ceftazidime MICs of $n f x C$ mutants (Maseda et al., 2000). However, another study showed that the functional subunit of the mexEF-oprN operon is not related to beta-lactam hypersusceptibility (Maseda et al., 2000). These observations suggest the existence of other co-operating systems responsible for beta-lactam susceptibility or resistance in $n f x C$ mutants. This issue remains unresolved. 
Table 2. Antibiotic-resistance phenotypes and extrinsic beta-lactamases in strains examined

Seventeen strains were examined: strains R1-7, resistant wild-type; S1-10, susceptible wild-type. Strains were classed as resistant (R) or susceptible (S) according to NCCLS criteria.

\begin{tabular}{|lcccccc|}
\hline Strain & Imipenem & Meropenem & Ceftazidime & Ciprofloxacin & Amikacin & Beta-lactamase $^{*}$ \\
\hline S1-S10 & S & S & S & S & S & None \\
R1 & S & R & R & R & R & None \\
R2 & R & S & S & S & S & None \\
R3 & R & S & S & R & S & None \\
R4 & R & R & R & S & S & oxa-17 \\
R5 & R & R & R & R & R & oxa-17 \\
R6 & R & R & R & S & R & pI 5·2 \\
R7 & R & R & R & S & R & pI 5·2 \\
\hline
\end{tabular}

* Other than chromosomal AmpC beta-lactamase.

$†$ From sequence analysis.

Table 3. MICs of ciprofloxacin-selected ( $n f x C$-like) mutants compared with wild-types

MICs are given in $\mu \mathrm{g} \mathrm{ml}^{-1}$ for the parent strain/mutant.

\begin{tabular}{|lccccc|}
\hline Mutant & Meropenem & Imipenem & Ceftazidime & Ciprofloxacin & Levofloxacin \\
\hline NFX1 & $0 \cdot 06 / 1$ & $0 \cdot 25 / 1$ & $0 \cdot 25 / 0 \cdot 25$ & $4 / 16$ & $8 / 32$ \\
NFX2 & $0 \cdot 5 / 1$ & $0 \cdot 25 / 1$ & $0 \cdot 25 / 4$ & $4 / 16$ & $8 / 64$ \\
NFX3 & $0 \cdot 12 / 0 \cdot 5$ & $0 \cdot 5 / 2$ & $4 / 0 \cdot 25$ & $4 / 32$ & $8 / 64$ \\
NFX4 & $0 \cdot 5 / 1$ & $0 \cdot 5 / 2$ & $0 \cdot 25 / 0 \cdot 125$ & $4 / 32$ & $8 / 64$ \\
\hline
\end{tabular}

The housekeeping metabolism of prokaryotes has been shown to be highly variable (Vandecasteele et al., 2001), so genes expressed stably under one condition might not be so under others. Stability in terms of mRNA expression in prokaryotic cells, therefore, should be tested under equivalent conditions with the investigated setting. In other words, internal controls intended for use in resistance gene quantification studies should be tested in strains with changing resistance phenotypes. The diversity in the resistance phenotypes of this study group fulfils this requirement.

Six genes were compared in this study group. In order to avoid co-regulated genes, we carefully selected genes that are distantly related in metabolic function and chromosomal order. The selected genes participate in critical functions. $p b p-2$ has a central role in peptidoglycan metabolism, while it has some relation to the rod-shape-determining protein. ampC is involved in cell-wall recycling (Jacobs et al., 1995). The sigma factor-encoding $r p o D$ is a critical housekeeping gene (Schnider et al., 1995). proC is involved in amino acid biosynthesis, while $f a b D$ is involved in a different class of metabolic function (Kutchma et al., 1999). However, their metabolic importance was not the only reason for selecting these genes. Equally important is that they all were shown in an Escherichia coli DNA array study to be expressed at levels comparable to outer-membrane proteins (Wei et al., 2001).
These data led us to assume that these genes might also be expressed in sufficient quantities in $P$. aeruginosa, a further advantage in optimization of the PCR test.

The raw quantities of mRNA of the six genes studied obtained by RT-PCR are shown in Table 4. Correlation coefficients indicated $p r o C$ and $r p o D$ as the most significant pair $(r=0.958 ; P<0 \cdot 001)$. Similarly, the stepwise exclusion of the genes with the highest $\mathrm{M}$ values by geNorm left proC $(\mathrm{M}=0 \cdot 36)$ and $\operatorname{rpoD}(\mathrm{M}=0 \cdot 36)$ as the most stable genes.

Next, the levels of oprD, oprM and oprN mRNA of the four $n f x C$-like isogenic mutants were compared with those of their wild-type counterparts. In this experiment, the geometric mean of the levels of proC and $r p o D$ in a sample was its normalization factor. Comparison of the normalized quantitative values of these genes is shown in Table 5 . The mean level of $\operatorname{opr} N$ was 1.3 times higher in the mutants, while the mean level of oprD was 1.28 times lower. Values were comparable for all the mutants. Interestingly, the statistical comparison was significant only for oprN concentrations.

Data on expression of these resistance genes in the literature have to date been obtained by immunoblotting. Evaluations were dependent on crude differences and lacked precise numerical values. Therefore, comparison of the results of this study with the literature was not possible. Moreover, immunoblotting indicates differences in the level of mature 
Table 4. Raw concentrations of mRNA of six genes from 23 strains obtained by RT-PCR

Concentrations are given as copies $\mu \mathrm{l}^{-1}\left(\times 10^{-3}\right)$. S1-S10 are susceptible strains; S5 and S6 are the parents of M1 and M2 (ciprofloxacin-selected non- $n f x C$-type mutants) and S7-S10 are the parents of NFX1-NFX4 (ciprofloxacin-selected $n f x C$-type mutants). R1-R7 are wild-type resistant isolates.

\begin{tabular}{|c|c|c|c|c|c|c|}
\hline Strain & proC & $f a b D$ & rpoD & rpos & $p b p-2$ & $\operatorname{ampC}$ \\
\hline S1 & $2 \cdot 17$ & $2 \cdot 28$ & $3 \cdot 32$ & $2 \cdot 56$ & $0 \cdot 37$ & $2 \cdot 40$ \\
\hline S2 & 0.92 & 0.047 & 1.33 & $1 \cdot 32$ & $0 \cdot 36$ & 1.62 \\
\hline S3 & $0 \cdot 64$ & 1.05 & $0 \cdot 76$ & $0 \cdot 77$ & $0 \cdot 43$ & $0 \cdot 53$ \\
\hline S4 & $1 \cdot 49$ & 1.52 & $2 \cdot 60$ & 1.92 & 0.49 & 1.74 \\
\hline S5 & $0 \cdot 35$ & $0 \cdot 29$ & $0 \cdot 30$ & $0 \cdot 51$ & 0.069 & 0.54 \\
\hline S6 & $0 \cdot 74$ & $1 \cdot 39$ & $1 \cdot 72$ & $1 \cdot 25$ & $0 \cdot 17$ & 1.29 \\
\hline S7 & $0 \cdot 24$ & $0 \cdot 31$ & $0 \cdot 28$ & $0 \cdot 87$ & $0 \cdot 21$ & $0 \cdot 29$ \\
\hline S8 & $0 \cdot 27$ & $0 \cdot 43$ & $0 \cdot 37$ & $1 \cdot 14$ & 0.04 & 0.59 \\
\hline S9 & $0 \cdot 42$ & $0 \cdot 41$ & $0 \cdot 50$ & $0 \cdot 70$ & $0 \cdot 21$ & $0 \cdot 60$ \\
\hline S10 & $0 \cdot 14$ & $0 \cdot 25$ & $0 \cdot 41$ & $0 \cdot 45$ & $0 \cdot 14$ & $0 \cdot 39$ \\
\hline $\mathrm{R} 1$ & $5 \cdot 91$ & $3 \cdot 82$ & $8 \cdot 55$ & $6 \cdot 89$ & $0 \cdot 68$ & $7 \cdot 65$ \\
\hline $\mathrm{R} 2$ & $2 \cdot 42$ & 3.67 & $4 \cdot 79$ & $5 \cdot 20$ & $0 \cdot 329$ & $5 \cdot 60$ \\
\hline R3 & $0 \cdot 48$ & $0 \cdot 82$ & $0 \cdot 89$ & $0 \cdot 41$ & $0 \cdot 30$ & $1 \cdot 13$ \\
\hline $\mathrm{R} 4$ & 1.92 & $2 \cdot 00$ & $2 \cdot 51$ & $2 \cdot 41$ & $0 \cdot 17$ & $3 \cdot 11$ \\
\hline R5 & $1 \cdot 88$ & $1 \cdot 24$ & $2 \cdot 75$ & $2 \cdot 87$ & $0 \cdot 17$ & $2 \cdot 74$ \\
\hline R6 & $0 \cdot 96$ & 0.66 & $1 \cdot 40$ & $1 \cdot 18$ & $0 \cdot 13$ & 1.39 \\
\hline R7 & 0.38 & $0 \cdot 29$ & $0 \cdot 58$ & $0 \cdot 46$ & $0 \cdot 21$ & $0 \cdot 77$ \\
\hline M1 & $1 \cdot 50$ & $1 \cdot 31$ & $2 \cdot 26$ & 1.98 & $0 \cdot 24$ & $2 \cdot 58$ \\
\hline M2 & 1.60 & 1.66 & $2 \cdot 51$ & 1.94 & $1 \cdot 31$ & 1.39 \\
\hline NFX1 & 0.92 & 0.95 & $1 \cdot 17$ & $1 \cdot 26$ & $0 \cdot 48$ & $1 \cdot 50$ \\
\hline NFX2 & $0 \cdot 47$ & $0 \cdot 60$ & $0 \cdot 80$ & 0.93 & $0 \cdot 13$ & 1.04 \\
\hline NFX3 & $0 \cdot 87$ & $0 \cdot 86$ & 1.49 & $1 \cdot 22$ & $0 \cdot 56$ & $1 \cdot 42$ \\
\hline NFX4 & $1 \cdot 18$ & $1 \cdot 11$ & $1 \cdot 71$ & $1 \cdot 45$ & 0.92 & $1 \cdot 87$ \\
\hline
\end{tabular}

Table 5. Comparison of mRNA concentrations of $n f x C$-like mutants and their wild-type counterparts

\begin{tabular}{|lccc|}
\hline Property & $\begin{array}{c}\text { Wild-type } \\
(\boldsymbol{n}=4)\end{array}$ & $\begin{array}{c}\text { Mutants } \\
(\boldsymbol{n}=4)\end{array}$ & $\boldsymbol{P}^{\star}$ \\
\hline oprD & & & \\
Mean (SD) & $0 \cdot 883(0 \cdot 169)$ & $0.689(0 \cdot 104)$ & 0.099 \\
Geometric mean & $0 \cdot 872$ & 0.683 & \\
Variance & $0 \cdot 029$ & $0 \cdot 011$ & \\
oprM & & & \\
Mean (SD) & $1 \cdot 173(0 \cdot 173)$ & $1 \cdot 169(0.202)$ & 0.977 \\
Geometric mean & $1 \cdot 163$ & $1 \cdot 155$ & \\
Variance & 0.030 & $0 \cdot 041$ & \\
oprN & & & \\
Mean (SD) & $0 \cdot 899(0 \cdot 066)$ & $1.208(0.207)$ & 0.030 \\
Geometric mean & $0 \cdot 897$ & $1 \cdot 195$ & \\
Variance & 0.004 & 0.043 & \\
\hline
\end{tabular}

*Obtained by ANOVA.

protein. It is known that mRNA expression predicts mature protein levels poorly and there may be up to 30 -fold differences (Gygi et al., 1999). Transcription and translation are regulated individually to some extent. The levels of mRNA of these genes may, therefore, not be exactly in accord with protein levels. However, the quantification results with RT-PCR in this study were in agreement with the expected expression profile of $n f x C$-type mutants.

Resistance due to the altered regulation of intrinsic genes in $P$. aeruginosa is not well understood. This type of resistance may depend on the regulation of a more composite coregulated network of multiple operons as well as the 'quorum-sensing systems' of $P$. aeruginosa (Kohler et al., 2001; Poole, 2001). Unfortunately, our understanding of this is extremely limited due to the limited power of the methods used at present. Therefore, further studies using new methods deserve increased scientific interest. We believe that realtime quantification with the selection of suitable internal control genes will facilitate studies and provide new insights into the regulatory alterations of innate genes and the multiple antibiotic resistance problem of $P$. aeruginosa.

This study showed that proC and $r p o D$ form the most stable pair in a set of clonally unrelated $P$. aeruginosa strains with diverse resistance phenotypes. Thus, this pair may be used as internal controls in relative comparison studies of resistance genes in $P$. aeruginosa. 


\section{ACKNOWLEDGEMENTS}

We are grateful to Dr Serhat Unal (Hacettepe Universitesi), Dr Mehmet Ali Inal (Ege Universitesi) and Dr Hakan Leblebicioglu (Ondokuz Mayıs Universitesi) for providing strains and to Jason John Nash and Victor L. Yu for English reading of this manuscript.

\section{REFERENCES}

Gygi, S. P., Rochon, Y., Franza, B. R. \& Aebersold, R. (1999). Correlation between protein and mRNA abundance in yeast. Mol Cell Biol 19, 1720-1730.

Jacobs, C., Joris, B., Jamin, M. \& 7 other authors (1995). AmpD, essential for both beta-lactamase regulation and cell wall recycling, is a novel cytosolic $\mathrm{N}$-acetylmuramyl-L-alanine amidase. Mol Microbiol 15, $553-559$.

Johnson, M. R., Wang, K., Smith, J. B., Heslin, M. J. \& Diasio, R. B. (2000). Quantitation of dihydropyrimidine dehydrogenase expression by real-time reverse transcription polymerase chain reaction. Anal Biochem 278, 175-184.

Kerr, J. R., Moore, J. E., Curran, M. D., Graham, R., Webb, C. H., Lowry, K. G., Murphy, P. G., Wilson, T. S. \& Ferguson, W. P. (1995). Investigation of a nosocomial outbreak of Pseudomonas aeruginosa pneumonia in an intensive care unit by random amplification of polymorphic DNA assay. J Hosp Infect 30, 125-131.

Kohler, T., van Delden, C., Curty, L. K., Hamzehpour, M. M. \& Pechere, J. C. (2001). Overexpression of the MexEF-OprN multidrug efflux system affects cell-to-cell signaling in Pseudomonas aeruginosa. J Bacteriol 183, 5213-5222.

Kutchma, A. J., Hoang, T. T. \& Schweizer, H. P. (1999). Characterization of a Pseudomonas aeruginosa fatty acid biosynthetic gene cluster: purification of acyl carrier protein (ACP) and malonyl-coenzyme A : ACP transacylase (FabD). J Bacteriol 181, 5498-5504.

Livermore, D. M. (1992). Interplay of impermeability and chromosomal beta-lactamase activity in imipenem-resistant Pseudomonas aeruginosa. Antimicrob Agents Chemother 36, 2046-2048.

Maseda, H., Yoneyama, H. \& Nakae, T. (2000). Assignment of the substrate-selective subunits of the MexEF-OprN multidrug efflux pump of Pseudomonas aeruginosa. Antimicrob Agents Chemother 44, 658-664.

Masuda, N., Sakagawa, E., Ohya, S., Gotoh, N., Tsujimoto, H. \& Nishino, T. (2000). Substrate specificities of MexAB-OprM, MexCDOprJ, and MexXY-oprM efflux pumps in Pseudomonas aeruginosa. Antimicrob Agents Chemother 44, 3322-3327.

Morita, Y., Komori, Y., Mima, T., Kuroda, T., Mizushima, T. \& Tsuchiya, T. (2001). Construction of a series of mutants lacking all of the four major mex operons for multidrug efflux pumps or possessing each one of the operons from Pseudomonas aeruginosa PAO1: MexCD-OprJ is an inducible pump. FEMS Microbiol Lett 202, 139-143.

Ochs, M. M., McCusker, M. P., Bains, M. \& Hancock, R. E. (1999).
Negative regulation of the Pseudomonas aeruginosa outer membrane porin OprD selective for imipenem and basic amino acids. Antimicrob Agents Chemother 43, 1085-1090.

Okamoto, K., Gotoh, N. \& Nishino, T. (2001). Pseudomonas aeruginosa reveals high intrinsic resistance to penem antibiotics: penem resistance mechanisms and their interplay. Antimicrob Agents Chemother 45, 1964-1971.

Poole, K. (2001). Multidrug efflux pumps and antimicrobial resistance in Pseudomonas aeruginosa and related organisms. J Mol Microbiol Biotechnol 3, 255-264.

Renders, N., Romling, Y., Verbrugh, H. \& van Belkum, A. (1996). Comparative typing of Pseudomonas aeruginosa by random amplification of polymorphic DNA or pulsed-field gel electrophoresis of DNA macrorestriction fragments. J Clin Microbiol 34, 3190-3195.

Schnider, U., Keel, C., Blumer, C., Troxler, J., Defago, G. \& Haas, D. (1995). Amplification of the housekeeping sigma factor in Pseudomonas fluorescens $\mathrm{CHA} 0$ enhances antibiotic production and improves biocontrol abilities. J Bacteriol 177, 5387-5392.

Sumita, Y. \& Fukasawa, M. (1996). Meropenem resistance in Pseudomonas aeruginosa. Chemotherapy 42, 47-56.

Trias, J., Dufresne, J., Levesque, R. C. \& Nikaido, H. (1989). Decreased outer membrane permeability in imipenem-resistant mutants of Pseudomonas aeruginosa. Antimicrob Agents Chemother 33, 1202-1206.

Vahaboglu, H., Ozturk, R., Akbal, H., Saribas, S., Tansel, O. \& Coskunkan, F. (1998). Practical approach for detection and identification of OXA-10-derived ceftazidime-hydrolyzing extended-spectrum beta-lactamases. J Clin Microbiol 36, 827-829.

Vandecasteele, S. J., Peetermans, W. E., Merckx, R. \& Van Eldere, J. (2001). Quantification of expression of Staphylococcus epidermidis housekeeping genes with Taqman quantitative PCR during in vitro growth and under different conditions. J Bacteriol 183, 7094-7101.

Vandesompele, J., De Preter, K., Pattyn, F., Poppe, B., Van Roy, N., De Paepe, A. \& Speleman, F. (2002). Accurate normalization of realtime quantitative RT-PCR data by geometric averaging of multiple internal control genes. Genome Biol 3, research0034.1-0034.11. http:// genomebiology.com/2002/3/7/research/0034

Wang, T. \& Brown, M. J. (1999). mRNA quantification by real time TaqMan polymerase chain reaction: validation and comparison with RNase protection. Anal Biochem 269, 198-201.

Wei, Y., Lee, J. M., Richmond, C., Blattner, F. R., Rafalski, J. A. \& LaRossa, R. A. (2001). High-density microarray-mediated gene expression profiling of Escherichia coli. J Bacteriol 183, 545-556.

Yucesoy, M., Yulug, N., Kocagoz, S., Unal, S., Cetin, S. \& Calangu, S. (2000). Antimicrobial resistance of gram-negative isolates from intensive care units in Turkey: comparison to previous three years. J Chemother 12, 294-298. 